\title{
Segregated Pathways Carrying Frontally Derived Top-Down Signals to Visual Areas MT and V4 in Macaques
}

\author{
Taihei Ninomiya, ${ }^{1,2}$ Hiromasa Sawamura, ${ }^{1,3}$ Ken-ichi Inoue, ${ }^{1,2}$ and Masahiko Takada ${ }^{1,2}$ \\ ${ }^{1}$ Department of System Neuroscience, Tokyo Metropolitan Institute for Neuroscience, Tokyo 183-8526, Japan, ${ }^{2}$ Systems Neuroscience Section, Primate

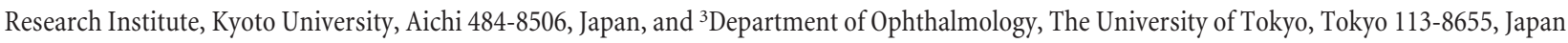

The bottom-up processing of visual information is strongly influenced by top-down signals, at least part of which is thought to be conveyed from the frontal cortex through the frontal eye field (FEF) and the lateral intraparietal area (LIP). Here we investigated the architecture of multisynaptic pathways from the frontal cortex to the middle temporal area (MT) of the dorsal visual stream and visual area 4 (V4) of the ventral visual stream in macaques. In the first series of experiments, the retrograde trans-synaptic tracer, rabies virus, was injected into MT or V4. Three days after rabies injections, the second-order (disynaptically connected) neuron labeling appeared in the ventral part of area 46 (area 46v), along with the first-order (monosynaptically connected) neuron labeling in FEF and LIP. In the MT-injection case, second-order neurons were also observed in the supplementary eye field (SEF). In the next series of experiments, double injections of two fluorescent dyes, fast blue and diamidino yellow, were made into MT and V4 to examine whether the frontal inputs are mediated by distinct or common neuronal populations. Virtually no double-labeled neurons were observed in FEF or LIP, indicating that separate neuronal populations mediate the frontal inputs to MT and V4. The present results define that the multisynaptic frontal input to V4 arises primarily from area 46v, whereas the input to MT arises from not only area 46v but also SEF, through distinct FEF and LIP neurons. Segregated pathways from the frontal cortex possibly carry the functionally diverse top-down signals to each visual stream.

\section{Introduction}

The primate visual system constructs parallel pathways known as dorsal and ventral streams. The two streams are functionally distinct in that the former is involved in action guidance, whereas the latter is related to object recognition, respectively (for review, see Maunsell and Newsome, 1987; Orban, 2008; Nassi and Callaway, 2009). Many studies have shown that top-down signals strongly modulate neuronal activities even in early visual areas that receive direct input from the primary visual cortex (V1). For example, neurons in the middle temporal area (MT) of the dorsal stream increase their activities when attention is directed within the receptive field (RF) (Treue and Maunsell, 1996, 1999; Ferrera and Lisberger, 1997; Seidemann and Newsome, 1999). In the ventral stream, neurons in visual area 4 (V4) filter irrelevant information from the RF (Moran and Desimone, 1985; Luck et al., 1997). It has also been shown that attentional effects are larger and earlier in V4 than V1 and visual area 2 (V2) (Mehta et al., 2000; Buffalo et al., 2010) and lesions in V4 affect the filtering of distracters at high resolution in downstream area TE (Buffalo et

\footnotetext{
Received Dec. 18, 2011; revised March 21, 2012; accepted March 23, 2012.

Author contributions: T.N., H.S., and M.T. designed research; T.N., H.S., and K.-i.l. performed research; T.N. analyzed data; T.N., H.S., K.-i.I., and M.T. wrote the paper.

This work was supported by Grants-in-Aid for Scientific Research on Priority Areas-Integrative Brain Research (20019011) to H.S., as well as Young Scientists (B) (19790162 and 22790202) to H.S. from the Ministry of Education, Culture, Sports, Science, and Technology of Japan.

Correspondence should be addressed to Masahiko Takada, Systems Neuroscience Section, Primate Research Institute, Kyoto University, 41-2 Kanrin, Inuyama, Aichi 484-8506, Japan. E-mail: takada@pri.kyoto-u.ac.jp.

DOI:10.1523/JNEUROSCI.6295-11.2012

Copyright $\odot 2012$ the authors $\quad 0270-6474 / 12 / 326851-08 \$ 15.00 / 0$
}

al., 2005). These findings suggest that V4 plays important roles in providing top-down signals to visual areas in the ventral stream.

The frontal cortex is one of the possible sources for modulatory signals to MT and V4, as neurons in the frontal cortex represent task-relevant information that is presumably necessary for such signals (Funahashi et al., 1990; Everling et al., 2006). However, most frontal areas do not send output directly to early visual areas. Accumulated evidence to date indicates that the frontal eye field (FEF) and the lateral intraparietal area (LIP) project directly to many visual areas (Huerta et al., 1987; Blatt et al., 1990) and have been implicated in the involvement with attentional mechanisms that are thought to cause the modulation of neuronal activities in extrastriate visual areas including MT and V4 (Hopfinger et al., 2000; Kastner and Ungerleider, 2000; Corbetta and Shulman, 2002; Bisley and Goldberg, 2003; Moore and Armstrong, 2003; Wardak et al., 2004; Armstrong et al., 2006; Ekstrom et al., 2008). On the other hand, FEF and LIP have connections with multiple frontal areas (Blatt et al., 1990; Schall et al., 1995). It remains unclear where in the frontal cortex top-down signals are generated, or how such signals are conveyed to MT and V4.

In the present study, we investigated the architecture of frontally derived top-down signals conveyed toward MT and V4 through FEF or LIP. In the first series of experiments, retrograde transneuronal labeling with rabies virus was used to explore possible multisynaptic frontal inputs to MT and V4. In the second series of experiments, two fluorescent dyes, fast blue (FB) and diamidino yellow (DY), were injected into MT and V4 of single monkeys. Distributions of labeled neurons were analyzed in FEF and LIP to elucidate whether the frontal inputs to MT and V4 are 
mediated by distinct or common neuronal populations in these areas where the top-down signals are conveyed.

\section{Materials and Methods}

Tracer experiments using rabies virus and the fluorescent dyes were performed in the Tokyo Metropolitan Institute for Neuroscience (TMIN; Fuchu, Tokyo, Japan) or in the Primate Research Institute (PRI; Inuyama, Aichi, Japan) of Kyoto University, respectively. The experimental protocols were approved by the Animal Care and Use Committee of the TMIN and the Ethics Committee of the PRI. All experiments were conducted in accordance with the Tokyo Metropolitan Institute for Neuroscience Guidelines for the Care and Use of Animals (2000) by the TMIN and the Guide for Care and Use of Laboratory Primates by the PRI.

Six adult Japanese monkeys (Macaca fuscata) of either sex weighing $4-8.5 \mathrm{~kg}$ were used in this study. Details of the procedures for surgery, electrophysiological mapping, tracer injections, and histology were described previously (Ninomiya et al., 2011). Two additional monkeys with the $4 \mathrm{~d}$ survival period were also available as shown in the previous study, but data obtained in these monkeys were only briefly referred to in the present study for the following reasons. First, the distribution of trisynaptically connected neurons could not be compared in the MT- or V4injection cases, since no rabies injections were performed into MT with the $4 \mathrm{~d}$ survival period. Second, neuronal labeling was observed in a variety of areas in the frontal cortex on day 4 after the rabies injections into V4. The explosive increase in labeled areas indicated the existence of multiple bypass routes from the frontal cortex to V4 in a trisynaptic manner. We could not reasonably identify each of these routes.

Surgical procedures. Before rabies injections, a surgical operation for electrophysiological mapping was performed in each of the four monkeys. Under general anesthesia with ketamine hydrochloride $[10 \mathrm{mg} / \mathrm{kg}$ body weight (b.wt.), i.m.] and sodium pentobarbital ( $25 \mathrm{mg} / \mathrm{kg}$ b.wt., i.v.), a head holder were implanted under aseptic conditions. After several weeks of recovery and training periods, the monkeys were anesthetized with ketamine hydrochloride $(10 \mathrm{mg} / \mathrm{kg}$ b.wt., i.m.) and xylazine hydrochloride $(0.5-1 \mathrm{mg} / \mathrm{kg}$ b.wt., i.m.) and positioned in a stereotaxic frame attached to a monkey chair. A recording chamber was placed after a craniotomy, guided by stereotaxic coordinates of MT or V4.

Viral and tracer injections. In the case of rabies injections, electrophysiological mappings were primarily performed to evaluate the retinotopic positions of injection sites precisely. The challenge-virus-standard (CVS11) strain of rabies virus was injected into MT or V4 regions representing the desired eccentricity. The virus was originally obtained from the Centers for Disease Control and Prevention (Atlanta, GA) and was donated by Dr. Satoshi Inoue (National Institute of Infectious Diseases, Tokyo, Japan). This strain was the same as that introduced by Ugolini (1995) and Kelly and Strick (2000) to demonstrate specific retrograde trans-synaptic transport of the virus. The rate of retrograde transport for the viral batch used in this study was calibrated in our previous study (Miyachi et al., 2005). By evaluating transneuronal labeling in the cortico-basal and cerebro-cerebellar loop circuits, we concluded that it takes $\sim 2 \mathrm{~d}$ for first-order neuron labeling and one additional day per one synapse for subsequent transneuronal labeling. Moreover, the laminar distribution of neuronal labeling in V1 was analyzed in our recent study to confirm consistency with other studies on the same visual system by means of rabies virus (Nassi and Callaway, 2006; Nassi et al., 2006; Ninomiya et al., 2011). The titer of a viral suspension was $1.4 \times 10^{8}$ focus-forming units/ $\mathrm{ml}$. The viral suspension was injected by pressure through a $10 \mu \mathrm{lmod}-$ ified Hamilton microsyringe that allows us to monitor neuronal activities immediately before the injection (Tokuno et al., 1998). Three penetrations were typically made into MT or V4 at least $1 \mathrm{~mm}$ apart from each other. For each penetration, $0.5-0.75 \mu \mathrm{l}$ of rabies virus was deposited at two different depths (1-1.5 mm apart). In the case of FB and DY injections, a 0.2 tesla magnetic resonance scanner (General Electronic) was used to obtain a full coronal series of 1-mm-thick images for each monkey. Resulting structural images were used to calculate stereotaxic coordinates for the injections. Pressure injections of $4 \%$ FB (Illing), or 4\% DY (Illing) were made through a $10 \mu$ l Hamilton microsyringe. Two and four penetrations were made into MT or V4, respectively, at least $1 \mathrm{~mm}$ apart from each other. For each penetration, $0.5-0.75 \mu l$ of FB or DY was deposited at two different depths (1-1.5 $\mathrm{mm}$ apart).

Histological procedures and data analysis. Three days after the rabies injections or 3-4 weeks after the double injections of FB and DY, the monkeys were deeply anesthetized with an overdose $(50 \mathrm{mg} / \mathrm{kg}$ b.wt., i.v.) of sodium pentobarbital for perfusion-fixation. The monkeys were transcardially perfused with PBS (0.1 M, pH 7.4), followed by fixatives. The fixative for the monkeys with the rabies injections was a mixture of $10 \%$ formalin and $15 \%$ saturated picric acid in phosphate buffer $(\mathrm{PB} ; 0.1 \mathrm{M}$, $\mathrm{pH} 7.4$ ) and the one for the monkeys with FB and DY injections was $10 \%$ formalin in $0.1 \mathrm{M}$ PB. The brains were removed from the skull, postfixed in the same fresh fixative overnight, and saturated with $30 \%$ sucrose. Coronal or parasagittal sections were cut serially at $60 \mu \mathrm{m}$ thickness on a freezing microtome. In the case of rabies injections, a series of every sixth section was immunohistochemically stained for rabies virus with the standard avidin-biotin peroxidase complex (ABC) method as described previously (Miyachi et al., 2005; Ninomiya et al., 2011). Briefly, the sections were immersed in $1 \%$ skim milk for $1 \mathrm{~h}$ and incubated overnight at $4^{\circ} \mathrm{C}$ with rabbit anti-rabies antibody (diluted at 1:10,000; Inoue et al., 2003 ) in $0.1 \mathrm{M}$ PBS containing $0.1 \%$ Triton X-100 and $1 \%$ normal goat serum. The sections were then incubated for $2 \mathrm{~h}$ in the same fresh medium containing biotinylated goat anti-rabbit IgG antibody (diluted at 1:200; Vector Laboratories) and reacted with the ABC Elite kit (Vector Laboratories) for $1.5 \mathrm{~h}$. For visualization of the antigen, the sections were reacted in $0.05 \mathrm{M}$ Tris- $\mathrm{HCl}$ buffer containing $0.04 \% \mathrm{DAB}, 0.04 \%$ nickel chloride, and $0.002 \%$ hydrogen peroxide. These sections were counterstained with Neutral red. A series of adjacent sections was myelin-stained to visualize laminar or areal borders.

Images of sections for the rabies injections were digitally captured by bright-field microscopy (Biorevo) and imported into Adobe Illustrator to plot labeled neurons. After plotting of labeled neurons, the Matlab (The MathWorks) algorithm was used to calculate the number and percentage of neurons in each layer. Positions of FB- and DY-positive neurons in a series of every sixth section were plotted with Neurolucida software (MicroBrightField). Two series of adjacent sections were Nisslstained with cresyl violet and myelin-stained with a modified Gallyas method (Pistorio et al., 2006).

\section{Results}

\section{Injection sites of rabies virus in MT and V4}

Four monkeys received multiple injections of rabies virus into MT or V4 after electrophysiological identification. Details of the injection sites have been described previously (Ninomiya et al., 2011). It should be noted here that monkeys MT-a, MT-b, V4-a, and V4-b in the present study correspond to monkeys MT-3a, MT-3c, V4-3a, and V4-3b in this previous work, respectively. Only a brief account is provided here. In the case of MT injections, RFs around the injection sites for monkeys MT-a and MT-b ranged from eccentricities of $\sim 15-25^{\circ}$ or $10-20^{\circ}$, respectively. In both monkeys, RFs were located in the lower visual quadrant with a bias toward the horizontal meridian. In the case of V4 injections, RFs around the injection sites for monkeys V4-a and V4-b were in the lower visual quadrant at an eccentricity of $\sim 5-15^{\circ}$ and $15-40^{\circ}$, respectively. The retinotopic locations of neuronal labeling in V1 closely matched those in the injection sites for all cases. Such correspondence in the retinotopy between the injection sites and the distribution of labeled neurons in V1 indicate that the rabies injections were made properly into MT and V4.

\section{Distributions of retrogradely labeled neurons in frontal cortex after rabies injections into MT and V4}

Three days after the rabies injections into MT, substantial neuron labeling was observed in FEF (Fig. $1 D-F, J-L$ ), that is known to have direct connections with MT (Stanton et al., 1995). In both of monkeys MT-a and MT-b, labeled neurons were located mainly 
Monkey MT-a
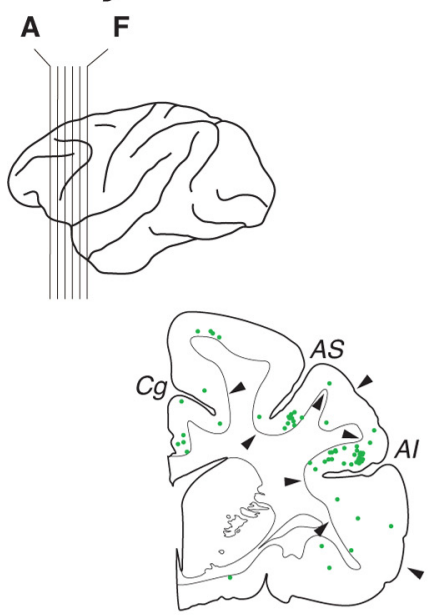

D

\section{Monkey MT-b}
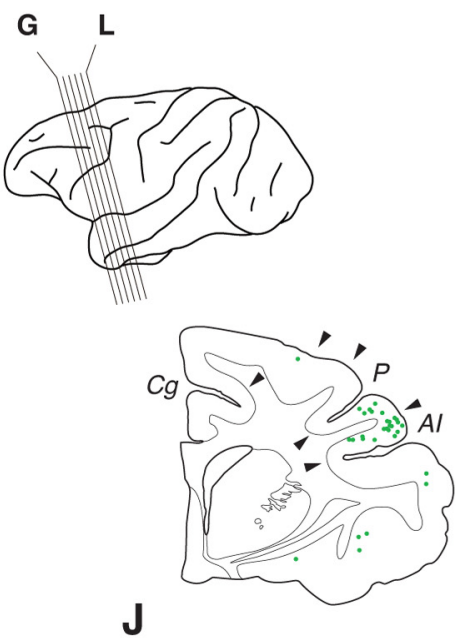

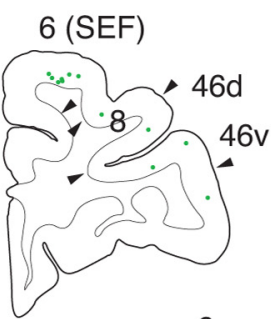

A

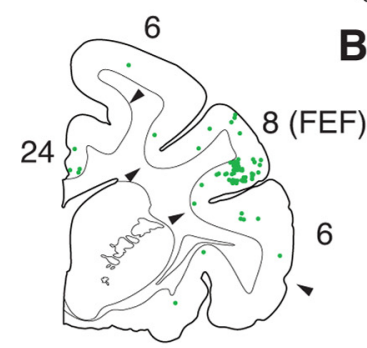

E

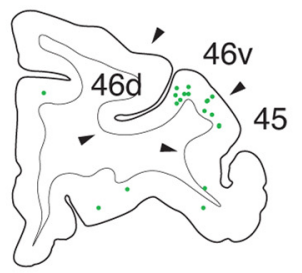

G

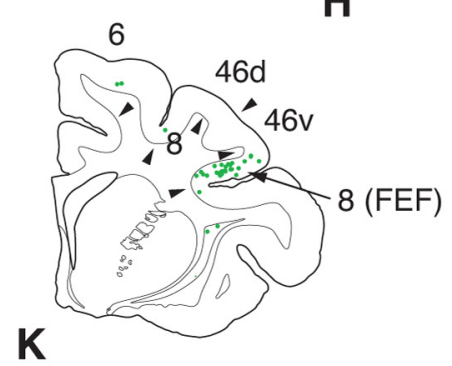

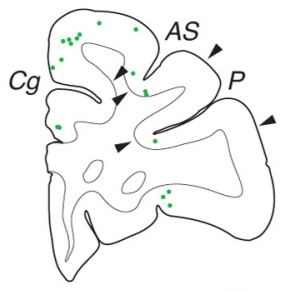

B

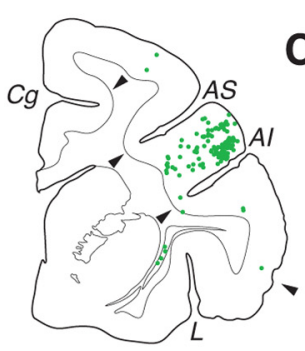

F

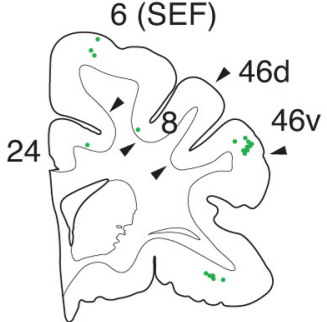

C

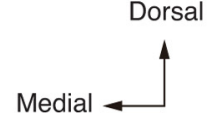

$10 \mathrm{~mm}$
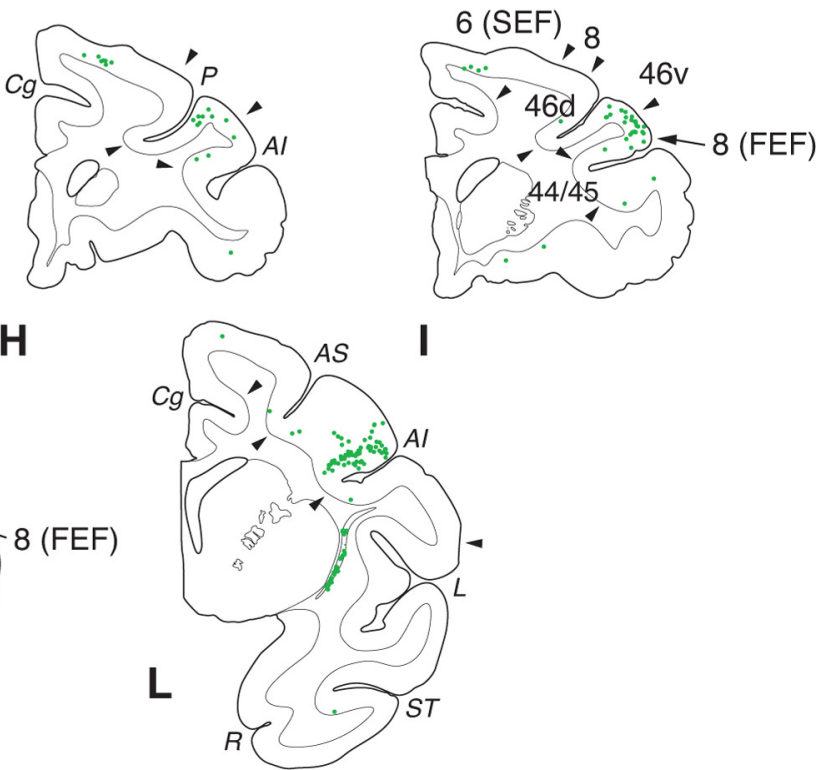

Figure 1. Distribution patterns of retrograde labeling in the frontal cortex $3 \mathrm{~d}$ after the rabies injections into MT. Six representative coronal sections for monkeys MT-a and MT-b are arranged anteroposteriorly in $\boldsymbol{A}-\boldsymbol{F}$ and $\mathbf{G}-\boldsymbol{L}$, respectively. The approximate anteroposterior levels of the sections are indicated in the lateral view of the brains. One green dot corresponds to one labeled neuron. Arrowheads indicate areal boarders. Areas and sulcus are depicted alternately in the sections for clarify. Al, Inferior limb of the arcuate sulcus; AS, superior limb of the arcuate sulcus; $\mathrm{Cg}$, cingulate sulcus; L, lateral sulcus; $P$, principal sulcus; $R$, rhinal sulcus; ST, superior temporal sulcus.

in the ventroposterior aspect of FEF. Previous studies have reported that the connectivity between FEF and MT has a topographic arrangement, and that the posterior part of FEF projects to the central visual field portion of MT (Barbas and Mesulam, 1981; Schall et at., 1995; Stanton et al., 1995). The distribution of rabies-labeled neurons in FEF was basically consistent with the results of these studies. Due to transneuronal transport of rabies virus, however, labeled neurons appeared even in the anterior aspect of FEF. Moreover, neuronal labeling was seen in the ventral part of area $46(46 \mathrm{v})$ and anteromedial part of area 6, corresponding to the supplementary eye field (SEF; Fig. $1 A-D, H, I$; Huerta and Kaas, 1990; Amiez and Petrides, 2009). Neuronal labeling in area $46 \mathrm{v}$ was located primarily around the lip of the principal sulcus (PS), corresponding to area $9 / 46 \mathrm{v}$ as designated in the study by Petrides and Pandya (1999). Neither area 46v nor SEF has direct connections with MT (Ungerleider and Desimone, 1986). We also confirmed the lack of direct connections between
MT and area 46v or SEF based on the results from FB injections into MT (monkeys Da and Db; data not shown). These overall findings suggest that multisynaptic inputs from the frontal cortex to MT are derived primarily from area $46 \mathrm{v}$ and SEF.

On day 3 after the rabies injections into V4, neuronal labeling occurred primarily in the ventroposterior aspect of FEF (Fig. $2 D-F, J-L)$. It has been revealed that $\mathrm{V} 4$ receives direct input from FEF in a topographic fashion that is similar to MT (Barbas and Mesulam, 1981; Schall et al., 1995; Stanton et al., 1995; Ungerleider et al., 2008). The distribution of rabies-labeled neurons in FEF was largely consistent with the data of the previous studies. Like the MT-injection cases, neuronal labeling was also observed in area 9/46v. Although FEF receives input from many areas in the frontal cortex including SEF, virtually no labeling was seen in other areas than area 46v and FEF. Previous studies have shown that area $46 \mathrm{v}$ does not directly project to V4 (Petrides and Pandya, 2002; Ungerleider et al., 2008), and again, the lack of a direct 


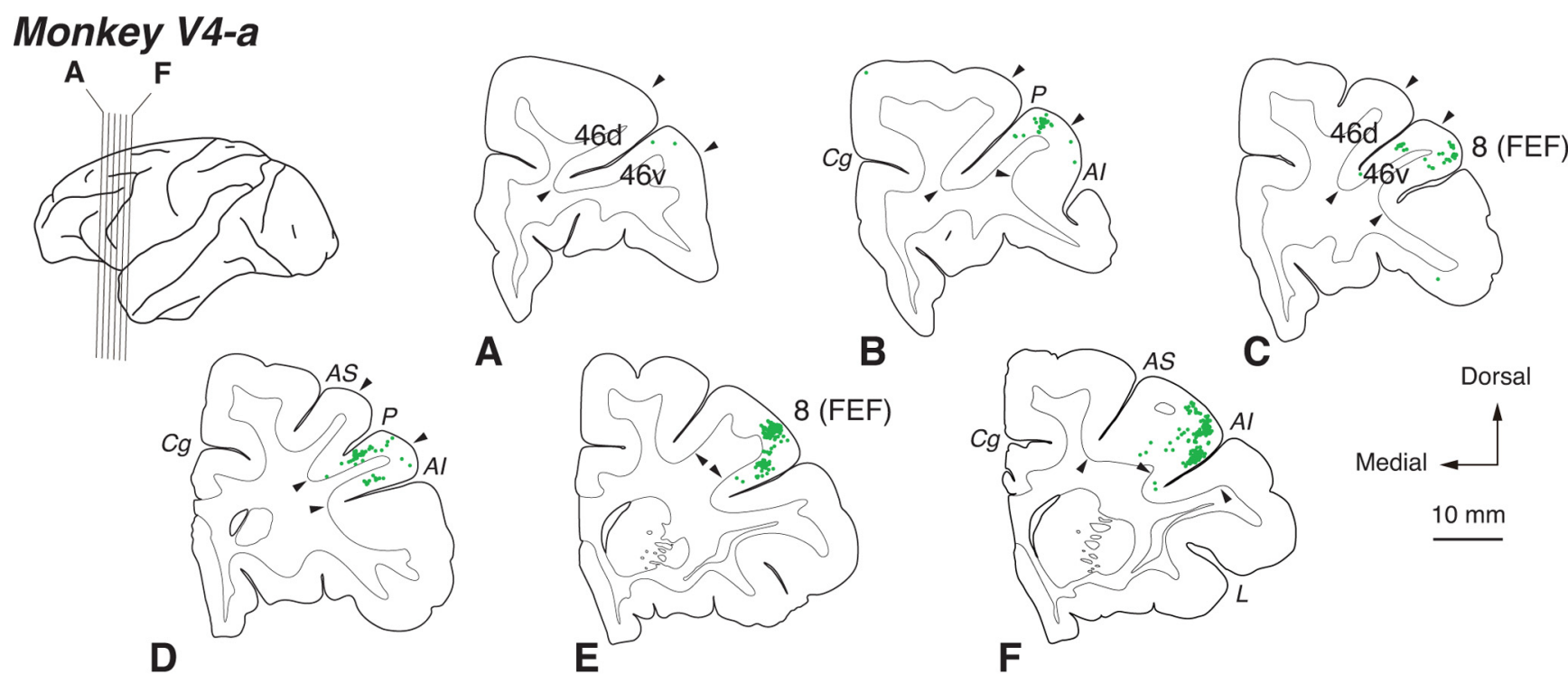

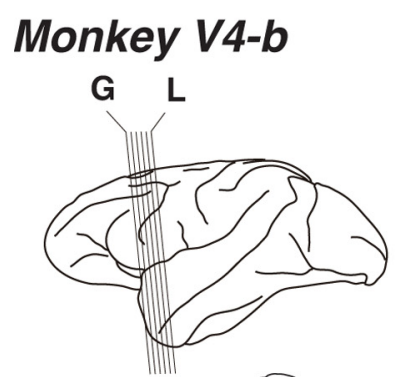

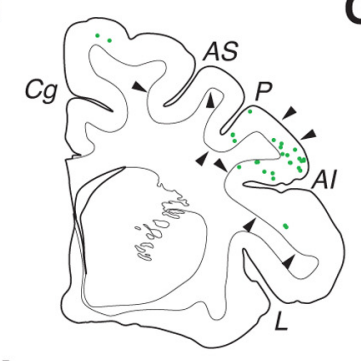

J

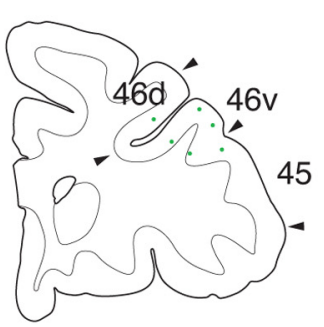

G

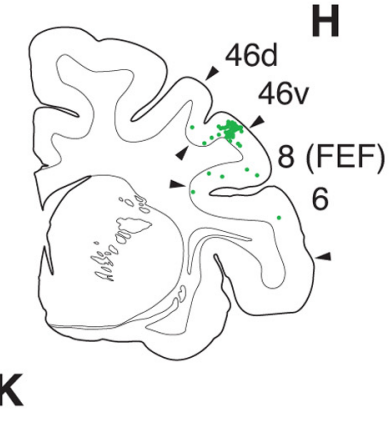

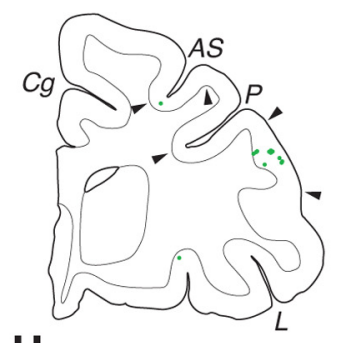
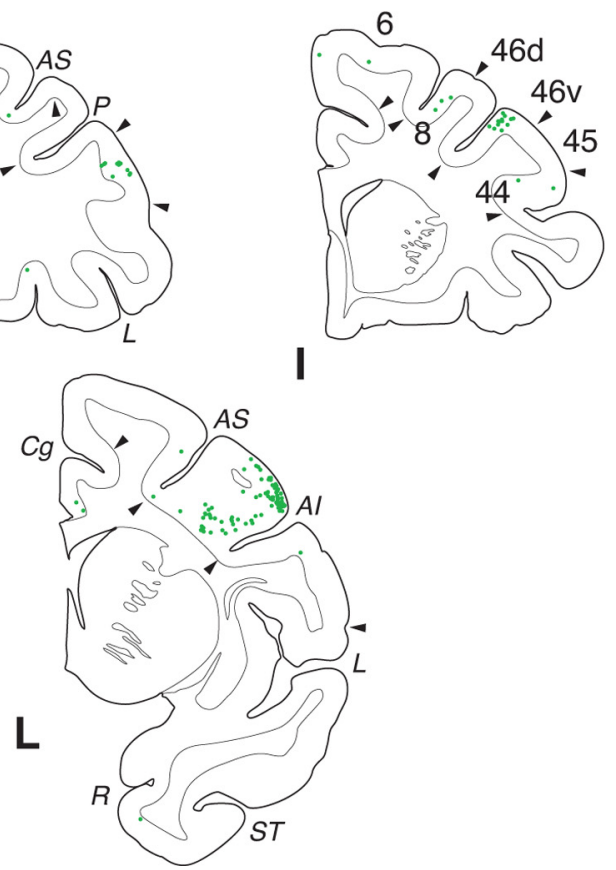

Figure 2. Distribution patterns of retrograde labeling in the frontal cortex $3 \mathrm{~d}$ after the rabies injections into V4. Six representative coronal sections for monkeys V4-a and V4-b are arranged anteroposteriorly in $\boldsymbol{A}-\boldsymbol{F}$ and $\mathbf{G}-\boldsymbol{L}$, respectively. For conventions, see Figure 1 .

connection between V4 and area $46 \mathrm{v}$ was confirmed with the results obtained from DY injections into V4 (monkeys Da and $\mathrm{Db}$; data not shown). The multisynaptic input from the frontal cortex to V4 seems to be derived primarily from area $46 \mathrm{v}$.

The distribution pattern of neuronal labeling in the frontal cortex was summarized in Figure 3. We counted the total number of labeled neurons as the number in the frontal lobe (including all areas anterior to the central sulcus) and calculated the percentage in each frontal area. In all injection cases, substantial labeling was observed in FEF (63.6\% for MT, 67.2\% for V4) and area $46 \mathrm{v}$ ( $18.3 \%$ for MT, $26.8 \%$ for V4). Additionally, neuronal labeling appeared in SEF for the MT-injection cases (9.1\%). A few labeled neurons were seen in areas 24 and the dorsal part of area 46 (46d) for the MT-injection cases, and in area 44/45 for both the MTand the V4-injection cases.
On day 4 after the rabies injections into V4, rabies-labeled neurons appeared in a variety of areas in the frontal cortex. In addition to FEF and area 46v, neuronal labeling was observed in the medial and lateral parts of area 9, and areas 11, 12, 24, 32, 44/45, 9/46d, 46d, 46v (anterior to area 9/46v), and SEF. The labeled neurons were seen predominantly in deep layers. In areas $12,44 / 45$, and SEF, dense neuron labeling was found in superficial and deep layers.

\section{Distributions of labeled neurons in FEF and LIP after double injections of FB and DY into MT and V4}

To assess whether the frontal inputs to MT and V4 are mediated by different or common neuronal populations, we injected FB and DY into retinotopically corresponding parts of MT or V4, respectively, in single monkeys (monkeys $\mathrm{Da}$ and $\mathrm{Db}$ ). The reti- 


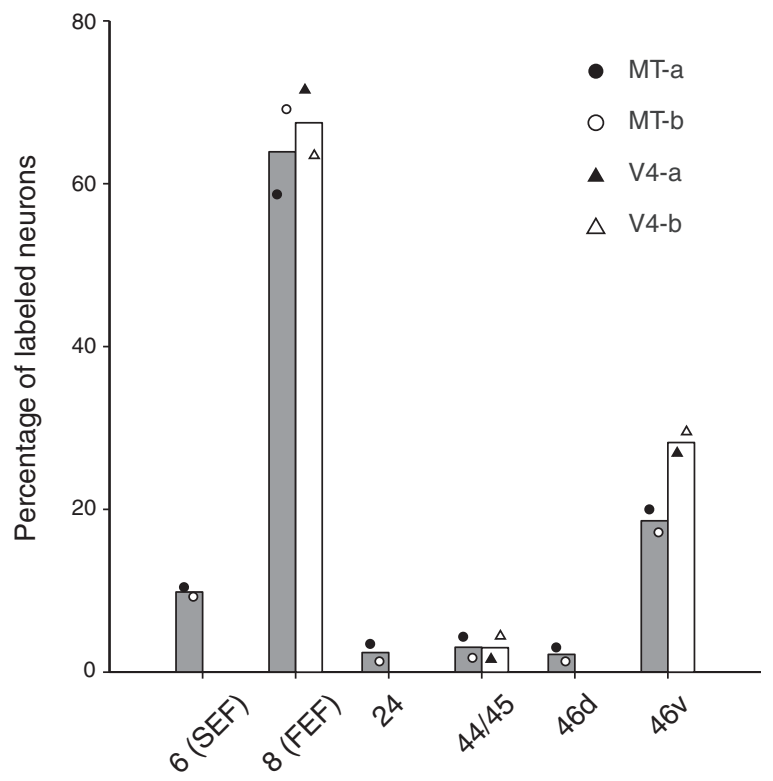

Figure 3. Summary histogram showing the distribution of neuronal labeling in the frontal cortex $3 \mathrm{~d}$ after the rabies injections into MT and V4. The percentage of labeled neurons in each injection case is specified by symbols indicated on the upper right of each panel. The diagrams in this figure include data only when the percentage of labeled neurons in each area exceeds $3 \%$. Each bar represents the averaged values of the MT (gray)- or V4 (white)-injection cases.

notopic extents of tracer injections in MT and V4 were evaluated by the distribution areas of FB- and DY-labeled neurons in V1 and V2 as well as by the extents of the injection sites. For monkey $\mathrm{Da}$, the approximate eccentricity of the injection site in MT was $5-35^{\circ}$ around the horizontal meridian with a bias toward the lower visual quadrant, whereas that of the injection site in V4 was $3-15^{\circ}$ in the lower visual quadrant. For monkey $\mathrm{Db}$, the location of the MT injections corresponded to the lower visual quadrant near the horizontal meridian at an eccentricity of $\sim 7-15^{\circ}$, whereas that of the V4 injections corresponded to the lower visual quadrant at an eccentricity of $\sim 5-25^{\circ}$.

Figure 4 shows the distribution patterns of FB- and DYpositive neurons in FEF and LIP (monkey Da), where signals from areas 46v and/or SEF are presumably relayed (see Discussion for details). In FEF, the FB-positive (MT-projecting) neurons were observed in both superficial and deep layers, whereas many of the DY-positive (V4-projecting) neurons were seen in superficial layers. On average, $59.7 \%$ and $75.3 \%$ of the FB- and DY-positive neurons were distributed in superficial layers (layers 2 and 3), respectively [FB, 59.6\% (152/255), and DY, 72.6\% (90/ 124) for Monkey Da; FB, 58.9\% (89/151), and DY, 80.0\% (56/70) for Monkey Db]. The other labeled neurons were observed in deep layers (layers 5 and 6). The laminar distributions of FEF neurons projecting to MT and V4 were basically consistent with previous findings (Barone et al., 2000; Palmer and Rosa, 2006; Pouget et al., 2009). Neuronal labeling in monkeys Da and Db was located mainly in the ventroposterior part of FEF where the central visual field portions in MT and V4 are known to receive inputs (Stanton et al., 1995; Ungerleider et al., 2008). In monkey $\mathrm{Da}, \mathrm{FB}$-positive neurons were further observed in the prearcuate gyrus immediately adjacent to the arcuate sulcus which has connections with more peripheral portions of the visual areas (Barbas and Mesulam, 1981; Stanton et al., 1995; Ungerleider et al., 2008). In LIP, FB- and DY-positive neurons were found predominantly in lower layers, with a limited number of DY-positive neurons in upper layers. On average, $88.1 \%$ and $61.4 \%$ of FB- and
DY-positive neurons were distributed in deep layers, respectively [FB, 93.6\% (58/62), and DY, 64.7\% (132/204) for Monkey Da; FB, 82.8\% (53/64), and DY, 59.5\% (201/338) for Monkey Db]. The other labeled neurons were seen in superficial layers. Although the distribution regions of FB- and DY-positive neurons in FEF and LIP were largely overlapped, only very rarely were neurons double-labeled with both dyes.

The summary histograms of the percentages of FB- and DYpositive neurons in FEF or LIP are shown in Figure 5. Very few neurons were double-labeled in either FEF or LIP $(<3 \%)$, suggesting that top-down signals from area $46 \mathrm{v}$ and/or SEF are conveyed to MT and V4 through different neuronal populations in FEF and LIP.

\section{Discussion}

In the present study, we investigated the architecture of multisynaptic frontal inputs to extrastriate visual areas MT and V4 by injections of the retrograde trans-synaptic tracer rabies virus or double injections of the fluorescent dyes FB and DY into MT and V4. In the first series of experiments, we found that numbers of labeled neurons appeared in area 46v, $3 \mathrm{~d}$ after the rabies injections, in addition to neuronal labeling in FEF, that has direct connections with MT and V4 (Stanton et al., 1995). Transneuronal labeling was also observed in SEF in the case of MT, but not $\mathrm{V} 4$, injections. In the second series of experiments, we demonstrated that only a small number of neurons were double-labeled in FEF and LIP, suggesting that distinct neuronal populations carry top-down signals from the frontal cortex to MT and V4. The present results indicate that the disynaptic frontal inputs to MT arise from area 46v and SEF, whereas those to V4 arise primarily from area $46 \mathrm{v}$ and that these top-down signals are relayed through distinct FEF and/or LIP neurons.

Considering that FEF receives inputs from many areas in the frontal cortex (Huerta et al., 1987), it is somewhat surprising that only one or two areas participate in the disynaptic projections from the frontal cortex to MT and V4. As neither area 46v nor SEF directly projects to MT or V4, neuronal labeling in these areas at the $3 \mathrm{~d}$ postinjection period verifies the trans-synaptic transport of rabies virus. Furthermore, the internal control tested in our laboratory (Miyachi et al., 2005) and the consistency of labeling patterns in V1 with previous data (Nassi and Callaway, 2006; Ninomiya et al., 2011) indicate that neuronal labeling in area $46 \mathrm{v}$ and SEF is ascribable to the second-order labeling occurring at the $3 \mathrm{~d}$ postinjection period. However, there may exist the possibility that some weak disynaptic connections remain unlabeled with the $3 \mathrm{~d}$ survival time. In fact, a small number of neurons were labeled in areas 25 and 45 after the MT injections and in area 44/45 after the MT or V4 injections. Nonetheless, the present results indicate that area $46 \mathrm{v}$ and SEF give rise to the major disynaptic frontal inputs to MT and V4, whereas these areas do not. As some studies have reported the topographic difference in the connectivity of dorsal (the large saccade part) and ventral (the small saccade part) FEF with extrastriate visual areas (Barbas and Mesulam, 1981; Schall et al., 1995; Stanton et al., 1995), it may be worth noting that our quantitative analysis produced the same results in some range of eccentricities for viral (MT, 10-25 ; V4, $\left.5-40^{\circ}\right)$ and fluorescent dye (MT, 5-35' $\mathrm{V} 4,3-25^{\circ}$ ) injections. In both series of experiments, however, labeled neurons appeared mainly in the ventroposterior part of FEF and the connectivity between dorsal FEF and MT or V4 is not fully examined in the present study.

Possible pathways linking the frontal cortex to MT and V4 are shown in Figure 6. As FEF has reciprocal connections with MT, 


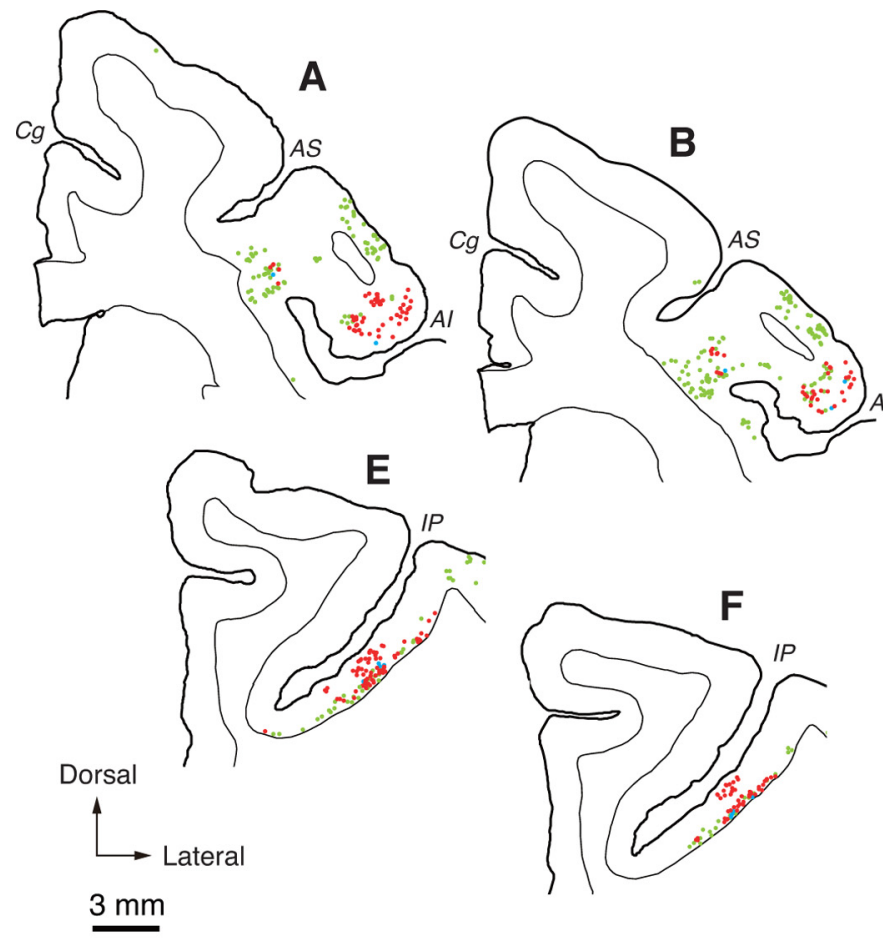

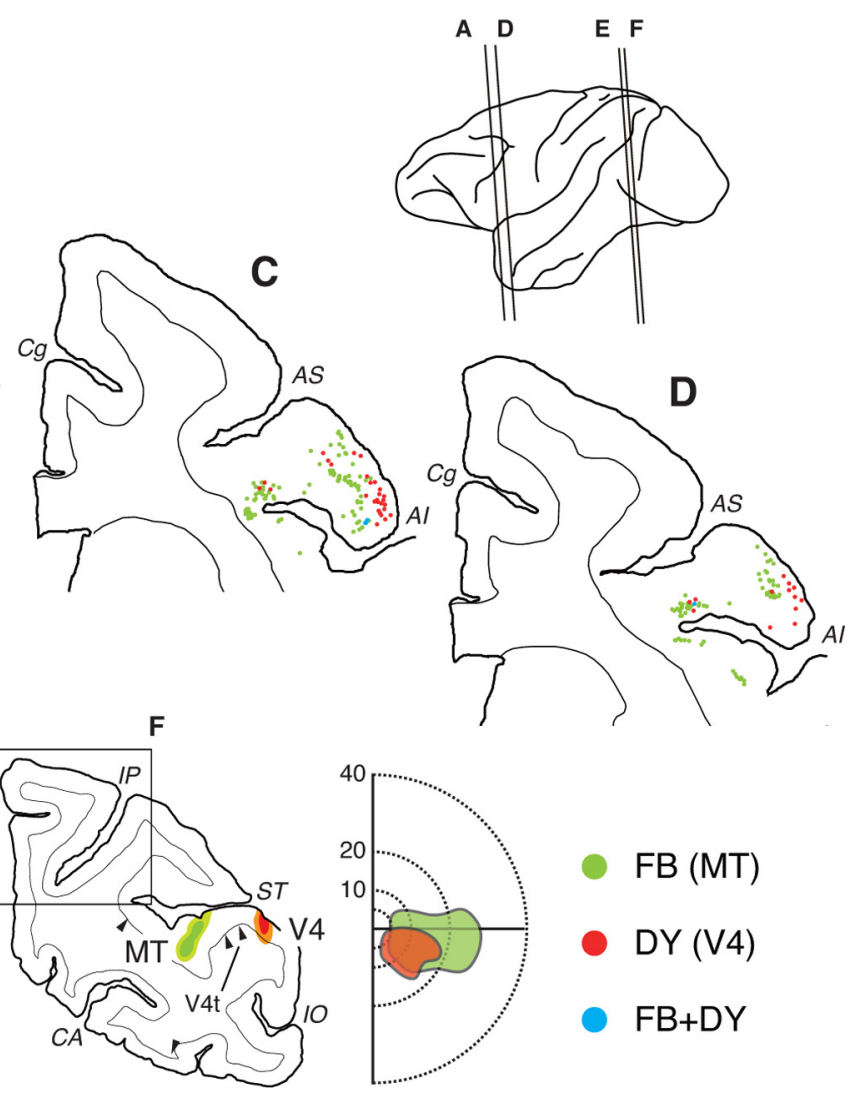

Figure 4. Distribution patterns of retrograde labeling in FEF and LIP after double injections of FB and DY into MT or V4 (monkey Da). Six representative coronal sections through FEF (A-D) or LIP $(\boldsymbol{E}, \boldsymbol{F})$ are arranged anteroposteriorly. The approximate anteroposterior levels of the sections are indicated in the lateral view of the brain. The extents of the injection sites of $F B$ (green) and DY (red) are exemplified in a coronal section at lower-right, part of which is enlarged in $\mathrm{F}$. The retinotopic extents of the injection sites are shown in an adjacent diagram of the contralateral hemifield (eccentricities denoted by numbers). Green dots, FB-positive neurons; red dots, DY-positive neurons; blue dots, double-labeled neurons. CA, Calcarine fissure; 10, inferior occipital sulcus; IP, intraparietal sulcus. Other abbreviations are as in Figure 1.

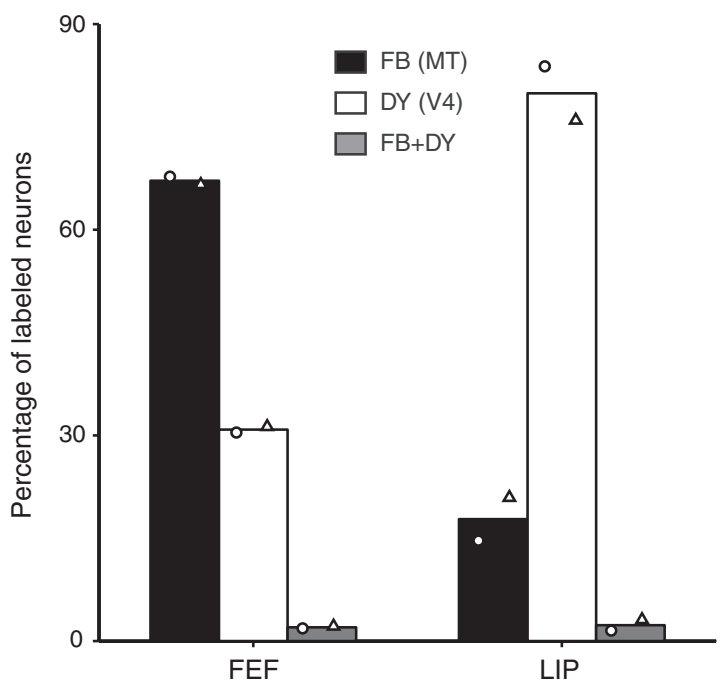

Figure 5. Summary histogram showing the percentage of FB- and DY-positive neurons in FEF or LIP. The percentage of each injection case is indicated by a circle (monkey $\mathrm{Da}$ ) and triangle (monkey Db). Each bar represents the averaged value of FB (black)-, DY (white)-, or doublelabeled (gray) neurons calculated from the two monkeys.

V4, area 46v, and SEF, it is most likely that this area mediates all multisynaptic inputs from the frontal cortex to MT and V4. Previous electrophysiological studies support the contribution of FEF in the top-down signals to extrastriate cortical areas, espe-

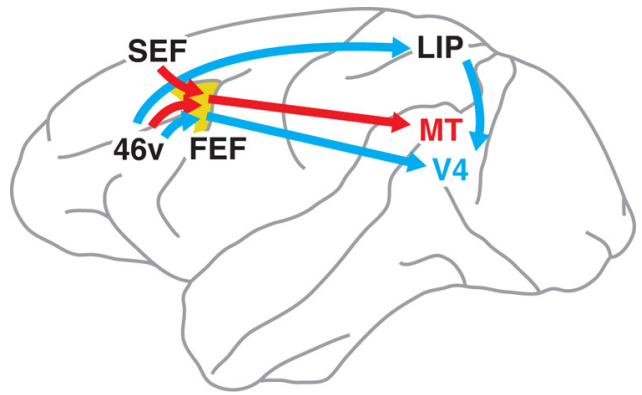

Figure 6. Organization of multisynaptic frontal inputs to MT and V4. MT presumably receives input from area $46 \mathrm{v}$ and SEF via FEF. In contrast, $\mathrm{V} 4$ likely receives input predominantly from area $46 \mathrm{v}$ via FEF. Also, LIP possibly relays signals from area $46 \mathrm{v}$ to $\mathrm{V} 4$. Only the most plausible pathways are shown in this schema (see Discussion for details).

cially V4. Microstimulation in FEF could modulate sensorydriven activity in early visual areas (Ekstrom et al., 2008), and even imitate the attentional modulation in V4 (Moore and Armstrong, 2003; Armstrong et al., 2006). The multisynaptic inputs may also be relayed via LIP, since LIP receives input directly from area 46v and sends output to V4 (Blatt et al., 1990; Barone et al., 2000; Lewis and Van Essen, 2000; Ungerleider et al., 2008). However, in view of the fact that LIP projects to layer 1 of MT only weakly (Blatt et al., 1990) and that SEF does not have an explicit projection to LIP (Blatt et al., 1990; Huerta and Kaas, 1990), it is less likely that LIP relays the disynaptic frontal inputs to MT. Other cortical areas, such as the medial superior temporal area 
(MST), the fundus of the superior temporal sulcus area (FST), ventral intraparietal area (VIP), and anterior intraparietal area (AIP), do not seem to participate in the frontal inputs to MT and V4, because MST, FST, and VIP do not receive input from the frontal cortex except for FEF (Boussaoud et al., 1990; Lewis and Van Essen, 2000). In addition, AIP has a connection with area $46 \mathrm{v}$, but does not send output to MT (Blatt et al., 1990). It has been reported that the inferotemporal cortex receives input from the ventral aspect of the frontal cortex, such as areas 8,12 , and 45 (Seltzer and Pandya, 1989; Distler et al., 1993; Webster et al., 1994). Considering the results of these studies, however, areas TE and TEO may receive input from the ventral lip of the PS that corresponds to the location of rabies-labeled neurons in the V4injection cases. Thus, areas TE and/or TEO may also relay signals from area $46 \mathrm{v}$ to $\mathrm{V} 4$.

Many studies clearly indicate that MT is closely related to oculomotor system (Komatsu and Wurtz, 1988a,b; Newsome et al., 1988; Chukoskie and Movshon, 2009). Using the transsynaptic tracing technique with rabies virus, Lyon et al. (2010) have recently shown that the superior colliculus (SC) sends robust output disynaptically to MT but not to V4. It has also been revealed in the present study that SEF, which is thought to play important roles in the oculomotor system, projects disynaptically to MT, but not to V4. Different laminar patterns of labeled neurons in FEF also suggest the functional difference in information conveyed to MT and V4. However, the present result as well as previous studies (Barone et al., 2000; Pouget et al., 2009) showed that FEF projects to MT and V4 mainly through superficial layers. This result implies that MT and V4 receive the FEF input related primarily to target selection (Thompson et al., 1996), which may give some implications for models of attention. For example, the premotor theory of attention (Craighero and Rizzolatti, 2005) hypothesizes that the same mechanism contributes to orienting attention and gaze shift. As electrical stimulation in FEF can modulate neuronal activity in MT and V4 (Moore and Armstrong, 2003; Armstrong et al., 2006; Ekstrom et al., 2008), the premotor theory predicts saccade command signals from FEF to MT and V4. This is inconsistent with the present result that the input from FEF arises mainly from superficial layers. Obviously pyramidal neurons in layer 5 of FEF, in which some of MT- and V4-projecting neurons exist, send movement and fixation signals to oculomotor-related areas like SC and the brainstem (Segraves and Goldberg, 1987; Segraves, 1992; Sommer and Wurtz, 2000). However, some neurons in the infragranular layer, including SCprojecting pyramidal neurons, are visually driven (Thompson et al., 1996; Sommer and Wurtz, 2000). It is thus possible that MT and $\mathrm{V} 4$ receive signals related only to target selection, but not to a saccade command. Although the present results as well as the study of Lyon et al. (2010) indicate the closer connectivity of MT with oculomotor-related areas, the nature of signals derived from FEF toward MT and V4 should be examined in terms of their relationships with target selection and saccadic eye movement.

The prefrontal cortex around the PS, often referred to as the dorsolateral prefrontal cortex (DLPFC), contains neurons that exhibit task-relevant responses to visual stimuli (Funahashi et al., 1990; Everling et al., 2006). Area 46v, where the second-order neuron labeling was observed in the present study, is among the DLPFC and may send task-relevant visual information to MT and V4. It is of interest that rabies-labeled neurons were located mostly in the ventral part of the PS, despite the existence of visually responsive neurons in both the dorsal and ventral parts (Funahashi et al., 1990; Everling et al., 2006). Hoshi and Tanji (2004) have reported that the ventral part of the DLPFC is involved in detecting or retaining spatial information contained in visual cues for future use, whereas the dorsal counter part participates in coding the target to reach or which arm to use for reaching behavior depending on a combination of two visual cues. This suggests that the information retaining in the ventral but not in the dorsal part of the DLPFC may be suitable for the source of topdown signals to MT and V4.

Together, the present results provide anatomical evidence that area $46 \mathrm{v}$ and SEF give rise to segregated multisynaptic pathways to MT and V4 by way of FEF and LIP. These pathways might contribute to the top-down mechanism in the frontal cortex that underlies visual information processing in the dorsal and ventral streams. Further studies will be necessary to elucidate the functional specificity of each pathway for better understanding of the role of the top-down signals in the parallel visual pathways.

\section{Notes}

Supplemental material for this article is available at http://www.pri.kyoto-u. ac.jp/sections/systems_neuroscience/publications.html\#sup. The distribution of labeled neurons in the frontal cortex $4 \mathrm{~d}$ after the rabies injections into $\mathrm{V} 4$ is provided as an online supplemental material on our website. This material has not been peer reviewed.

\section{References}

Amiez C, Petrides M (2009) Anatomical organization of the eye fields in the human and non-human primate frontal cortex. Prog Neurobiol 89:220-230.

Armstrong KM, Fitzgerald JK, Moore T (2006) Changes in visual receptive fields with microstimulation of frontal cortex. Neuron 50:791-798.

Barbas H, Mesulam MM (1981) Organization of afferent input to subdivisions of area 8 in the rhesus monkey. J Comp Neurol 200:407-431.

Barone P, Batardiere A, Knoblauch K, Kennedy H (2000) Laminar distribution of neurons in extrastriate areas projecting to visual areas V1 and V4 correlates with the hierarchical rank and indicates the operation of a distance rule. J Neurosci 20:3263-3281.

Bisley JW, Goldberg ME (2003) Neuronal activity in the lateral intraparietal area and spatial attention. Science 299:81-86.

Blatt GJ, Andersen RA, Stoner GR (1990) Visual receptive field organization and cortico-cortical connections of the lateral intraparietal area (area LIP) in the macaque. J Comp Neurol 299:421-445.

Boussaoud D, Ungerleider LG, Desimone R (1990) Pathways for motion analysis: cortical connections of the medial superior temporal and fundus of the superior temporal visual areas in the macaque. J Comp Neurol 296:462-495.

Buffalo EA, Bertini G, Ungerleider LG, Desimone R (2005) Impaired filtering of distracter stimuli by TE neurons following V4 and TEO lesions in macaques. Cereb Cortex 15:141-151.

Buffalo EA, Fries P, Landman R, Liang H, Desimone R (2010) A backward progression of attentional effects in the ventral stream. Proc Natl Acad Sci U S A 107:361-365.

Chukoskie L, Movshon JA (2009) Modulation of visual signals in macaque MT and MST neurons during pursuit eye movement. J Neurophysiol 102:3225-3233.

Corbetta M, Shulman GL (2002) Control of goal-directed and stimulusdriven attention in the brain. Nat Rev Neurosci 3:201-215.

Craighero L, Rizzolatti G (2005) The premotor theory of attention. In: Neurobiology of attention (Itti L, Rees G, Tsotsos JK, eds), pp 181-186. New York: Elsevier.

Distler C, Boussaoud D, Desimone R, Ungerleider LG (1993) Cortical connections of inferior temporal area TEO in macaque monkeys. J Comp Neurol 334:125-150.

Ekstrom LB, Roelfsema PR, Arsenault JT, Bonmassar G, Vanduffel W (2008) Bottom up dependent gating of frontal signals in early visual cortex. Science 321:414-417.

Everling S, Tinsley CJ, Gaffan D, Duncan J (2006) Selective representation of task-relevant objects and locations in the monkey prefrontal cortex. Eur J Neurosci 23:2197-2214.

Ferrera VP, Lisberger SG (1997) Neuronal responses in visual areas MT and MST during smooth pursuit target selection. J Neurophysiol 78:1433-1446. Funahashi S, Bruce CJ, Goldman-Rakic PS (1990) Visuospatial coding in 
primate prefrontal neurons revealed by oculomotor paradigms. J Neurophysiol 63:814-831.

Hopfinger JB, Buonocore MH, Mangun GR (2000) The neural mechanisms of top-down attentional control. Nat Neurosci 3:284-291.

Hoshi E, Tanji J (2004) Area-selective neuronal activity in the dorsolateral prefrontal cortex for information retrieval and action planning. J Neurophysiol 91:2707-2722.

Huerta MF, Kaas JH (1990) Supplementary eye field as defined by intracortical microstimulation: connections in macaques. J Comp Neurol 293:299-330.

Huerta MF, Krubitzer LA, Kaas JH (1987) Frontal eye field as defined by intracortical microstimulation in squirrel monkeys, owl monkeys, and macaque monkeys II. Cortical connections. J Comp Neurol 265:332-361.

Inoue S, Sato Y, Hasegawa H, Noguchi A, Yamada A, Kurata T, Iwasaki T (2003) Cross-reactive antigenicity of nucleoproteins of lyssaviruses recognized by a monospecific antirabies virus nucleoprotein antiserum on paraffin sections of formalin-fixed tissues. Pathol Int 53:525-533.

Kastner S, Ungerleider LG (2000) Mechanisms of visual attention in the human cortex. Annu Rev Neurosci 23:315-341.

Kelly RM, Strick PL (2000) Rabies as a transneuronal tracer of circuits in the central nervous system. J Neurosci Methods 103:63-71.

Komatsu H, Wurtz RH (1988a) Relation of cortical areas MT and MST to pursuit eye movements. I. Localization and visual properties of neurons. J Neurophysiol 60:580-603.

Komatsu H, Wurtz RH (1988b) Relation of cortical areas MT and MST to pursuit eye movements. III. Interaction with full-field visual stimulation. J Neurophysiol 60:621-644.

Lewis JW, Van Essen DC (2000) Corticocortical connections of visual, sensorimotor, and multimodal processing areas in the parietal lobe of the macaque monkey. J Comp Neurol 428:112-137.

Luck SJ, Chelazzi L, Hillyard SA, Desimone R (1997) Neural mechanisms of spatial selective attention in areas V1, V2, and V4 of macaque visual cortex. J Neurophysiol 77:24-42.

Lyon DC, Nassi JJ, Callaway EM (2010) A disynaptic relay from superior colliculus to dorsal stream visual cortex in macaque monkey. Neuron 65:270-279.

Maunsell JH, Newsome WT (1987) Visual processing in monkey extrastriate cortex. Annu Rev Neurosci 10:363-401.

Mehta AD, Ulbert I. Schroeder CE (2000) Intermodal selective attention in monkeys. I. Distribution and timing of effects across visual areas. Cereb Cortex 10:343-358.

Miyachi S, Lu X, Inoue S, Iwasaki T, Koike S, Nambu A, Takada M (2005) Organization of multisynaptic inputs from prefrontal cortex to primary motor cortex as revealed by retrograde transneuronal transport of rabies virus. J Neurosci 25:2547-2556.

Moore T, Armstrong KM (2003) Selective gating of visual signals by microstimulation of frontal cortex. Nature 421:370-373.

Moran J, Desimone R (1985) Selective attention gates visual processing in the extrastriate cortex. Science 229:782-784.

Nassi JJ, Callaway EM (2006) Multiple circuits relaying primate parallel visual pathways to the middle temporal area. J Neurosci 26:12789-12798.

Nassi JJ, Callaway EM (2009) Parallel processing strategies of the primate visual system. Nat Rev Neurosci 10:360-372.

Nassi JJ, Lyon DC, Callaway EM (2006) The parvocellular LGN provides a robust disynaptic input to the visual motion area MT. Neuron 50:319-327.

Newsome WT, Wurtz RH, Komatsu H (1988) Relation of cortical areas MT and MST to pursuit eye movements. II. Differentiation of retinal from extraretinal inputs. J Neurophysiol 60:604-620.

Ninomiya T, Sawamura H, Inoue K, Takada M (2011) Differential architec- ture of multisynaptic geniculo-cortical pathways to V4 and MT. Cereb Cortex 21:2797-2808.

Orban GA (2008) Higher order visual processing in macaque extrastriate cortex. Physiol Rev 88:59-89.

Palmer SM, Rosa MGP (2006) Quantitative analysis of the corticocortical projections to the middle temporal area in the marmoset monkey: evolutionary and functional implications. Cereb Cortex 16:1361-1375.

Petrides M, Pandya DN (1999) Dorsolateral prefrontal cortex: comparative cytoarchitectonic analysis in the human and the macaque brain and corticocortical connection patterns. Eur J Neurosci 11:1011-1036.

Petrides M, Pandya DN (2002) Comparative cytoarchitectonic analysis of the human and the macaque ventrolateral prefrontal cortex and corticocortical connection patterns in the monkey. Eur J Neurosci 16:291-310.

Pistorio AL, Hendry SH, Wang X (2006) A modified technique for highresolution staining of myelin. J Neurosci Methods 153:135-146.

Pouget P, Stepniewska I, Crowder EA, Leslie MW, Emeric EE, Nelson MJ, Schall JD (2009) Visual and motor connectivity and the distribution of calcium-binding proteins in macaque frontal eye field: implications for saccade target selection. Front Neuroanat 3:2.

Schall JD, Morel A, King DJ, Bullier J (1995) Topography of visual cortex connections with frontal eye field in macaque: convergence and segregation of processing streams. J Neurosci 15:4464-4487.

Segraves MA (1992) Activity of monkey frontal eye field neurons projecting to oculomotor regions of the pons. J Neurophysiol 68:1967-1985.

Segraves MA, Goldberg ME (1987) Functional properties of corticotectal neurons in the monkey's frontal eye field. J Neurophysiol 58:1387-1419.

Seidemann E, Newsome WT (1999) Effect of spatial attention on the responses of area MT neurons. J Neurophysiol 81:1783-1794.

Seltzer B, Pandya DN (1989) Frontal lobe connections of the superior temporal sulcus in the rhesus monkey. J Comp Neurol 281:97-113.

Sommer MA, Wurtz RH (2000) Composition and topographic organization of signals sent from the frontal eye field to the superior colliculus. J Neurophysiol 83:1979-2001.

Stanton GB, Bruce CJ, Goldberg ME (1995) Topography of projections to posterior cortical areas from the macaque frontal eye fields. J Comp Neurol 353:291-305.

Thompson KG, Hanes DP, Bichot NP, Schall JD (1996) Perceptual and motor processing stages identified in the activity of macaque frontal eye field neurons during visual search. J Neurophysiol 76:4040-4055.

Tokuno H, Ikeuchi Y, Nambu A, Akazawa T, Imanishi M, Hamada I, Hasegawa N (1998) A modified microsyringe for extracellular recording of neuronal activity. Neurosci Res 31:251-255.

Treue S, Maunsell JH (1996) Attentional modulation of visual motion processing in cortical areas MT and MST. Nature 382:539-541.

Treue S, Maunsell JH (1999) Effects of attention on the processing of motion in macaque middle temporal and medial superior temporal visual cortical areas. J Neurosci 19:7591-7602.

Ugolini G (1995) Specificity of rabies virus as a transneuronal tracer of motor networks: transfer from hypoglossal motoneurons to connected second-order and higher order central nervous system cell groups. J Comp Neurol 356:457-480.

Ungerleider LG, Desimone R (1986) Cortical connections of visual area MT in the macaque. J Comp Neurol 248:190-222.

Ungerleider LG, Galkin TW, Desimone R, Gattass R (2008) Cortical connections of area V4 in the macaque. Cereb Cortex 18:477-499.

Wardak C, Olivier E, Duhamel JR (2004) A deficit in covert attention after parietal cortex inactivation in the monkey. Neuron 42:501-508.

Webster MJ, Bachevalier J, Ungerleider LG (1994) Connections of inferior temporal areas TEO and TE with parietal and frontal cortex in macaque monkeys. Cereb Cortex 4:470-483. 